\title{
Self-Triggered Stabilization of Continuous Stochastic State-Feedback Controlled Systems
}

\author{
Ross P. Anderson, Dejan Milutinović, and Dimos V. Dimarogonas
}

\begin{abstract}
Event-triggered and self-triggered control, in which the time of update to the controls is based on either current or outdated sampled data, have recently been employed to reduce the computational load or resource consumption for distributed real-time control systems. In this work, we propose a self-triggered scheme for nonlinear controlled stochastic differential equations with additive noise terms. A self-triggering update condition is derived that guarantees stability in the $p$-th moment of the state distribution. We show that the length of the times between controller updates as computed from the proposed scheme is strictly positive and provide examples.
\end{abstract}

\section{INTRODUCTION}

In sampled-data control systems, samples of continuous signals are used to compute and update controllers. How frequently the system should sample data and update its control is an important issue that has a large impact on system performance and stability. In traditional setups, these actions are scheduled periodically, but this may be conservative and lead to unnecessary computational load or bandwidth usage, for example. Alternative approaches, namely event- and selftriggering, have recently gained considerable attention [113]. The core of this idea is as follows: assuming that a continuous-time feedback control system is input-to-state stable [14] with respect to measurement errors, a condition relating the system state to the time at which the control should be updated is derived such that a Lyapunov function is guaranteed to be decreasing. Since the times are based on the state, the task periods may be longer than those under a periodic control implementation [2].

Using an event-triggered control implementation, the system state is sampled when an error signal first exceeds a threshold that is based on the current state [1-3]. A number of works (see [4,5] and the references therein, for example) extend these concepts for various notions of stability and to lengthen the amount of time between task updates. Since an event-triggered implementation requires continuous monitoring of the system state, which may be impractical for some systems, another alternative, self-triggering, decides the next sampling time instant from sampled data [6-11].

Common to most of these works is a deterministic system model, but a few attempts at the stochastic case have

Ross P. Anderson is a Graduate Student of Applied Mathematics and Statistics, University of California, Santa Cruz, 1156 High St, Santa Cruz, CA 95064, USA andersonesoe.ucsc.edu

Dejan Milutinović is an Assistant Professor in the Department of Applied Mathematics and Statistics, University of California, Santa Cruz dejan@soe.ucsc.edu

Dimos V. Dimarogonas is an Assistant Professor in the Automatic Control Lab, KTH Royal Institute of Technology, Osquldas v. 10, SE-100 44 Stockholm, Sweden dimos@kth.se appeared in literature. In [11], a self-triggered $\mathcal{H}_{\infty}$ control was developed for linear systems with a state-dependent disturbance, and this was extended in [12] for an exogenous disturbance in $\mathcal{L}_{2}$ space. The robustness of a self-triggered control strategy to disturbances was analyzed in [9] for linear systems.

In this work, we develop a self-triggered scheme for stochastic control systems described by stochastic differential equations (SDEs) [15] with additive noise terms. For systems of this type, it may be difficult to predict the system state at a future time. As such, we rely on the dynamics of the moments of the state distribution to develop inequality constraints on the controller update times based on the previously-measured state. We find inequality conditions for the length of time between controller updates that guarantee $p$-moment stability $[16,17](p>0)$ of the SDE solution. To the best of our knowledge, this work is the first implementation of self-triggered control for stochastic differential equations.

This paper is organized as follows: In Section II, we introduce relevant notation and definitions and formulate the problem. Section III proposes a self-triggering scheme with strictly positive times between control updates, and Section IV provides numerical examples. Finally, Section V summarizes the results of this paper.

\section{PRELIMINARIES AND PROBLEM STATEMENT}

\section{A. Notation and Definitions}

A function $\gamma: \mathbb{R}^{+} \rightarrow \mathbb{R}^{+}$is of class $\mathcal{K}$ if it is continuous, strictly increasing, and $\gamma(0)=0$. It is of class $\mathcal{K}_{\infty}$ if, in addition, it is unbounded. A function $\beta: \mathbb{R}^{+} \times[0, \infty) \rightarrow \mathbb{R}^{+}$ is of class $\mathcal{K} \mathcal{L}$ if, for each fixed $t, \beta(x, t)$ is of class $\mathcal{K}$, and, for each fixed $x, \beta(x, t)$ is decreasing with $\beta(x, t) \rightarrow 0$ as $t \rightarrow \infty$. Let $|\cdot|$ denote the Euclidian norm $|x|=\sqrt{x^{\top} x}$.

We will consider control systems defined by stochastic differential equations of the form

$$
\mathrm{d} x(t)=f(x, u) \mathrm{d} t+g(x, u) \mathrm{d} w, \quad x \in \mathbb{R}^{n},
$$

where $f(\cdot, \cdot)$ admits a fixed point $f(0,0)=0, \mathrm{~d} w$ is a (multi-dimensional) increment of a standard Wiener process, $u(t):[0, \infty) \rightarrow \mathbb{R}^{m}$ is a control input, and $f(\cdot)$ and $g(\cdot)$ are the drift and diffusion scaling factors of the dynamics. The differential operator $\mathcal{L}$ associated with a system in this form, when applied to a function $V(x, t)$ that is twice-differentiable in its first argument, is

$$
\mathcal{L} V(x, t)=\frac{\partial V}{\partial t}+f^{\top} \frac{\partial V}{\partial x}+\frac{1}{2} \operatorname{Trace}\left(g^{\top} \frac{\partial^{2} V}{\partial x^{2}} g\right) .
$$


Definition II.1 (cf. [18]). A system is said to be $p$-moment ${ }^{1}$ input-to-state stable (ISS) with respect to an error $e(t)$ if there exist a class $\mathcal{K} \mathcal{L}$ function $\beta$ and class $\mathcal{K}$ functions $\gamma$ and $\lambda$ such that for all $t \geq 0$,

$$
\mathbb{E}\left(|x(t)|^{p}\right) \leq \beta\left(\mathbb{E}\left(|x(0)|^{p}\right), t\right)+\gamma\left(\mathbb{E}\left(\lambda\left(\sup _{t \geq 0}|e(t)|\right)\right)\right) .
$$

If $\lambda(|e(t)|)$ is constant in time, we may also consider the following definition.

Definition II.2 (cf. [18]). A system is said to be practically $p$-moment stable if there exist a class $\mathcal{K} \mathcal{L}$ function $\beta$, a class $\mathcal{K}$ function $\gamma$, and a constant $d \geq 0$ such that for all $t \geq 0$,

$$
\mathbb{E}\left(|x(t)|^{p}\right) \leq \beta\left(\mathbb{E}\left(|x(0)|^{p}\right), t\right)+\gamma(d) .
$$

If $d=0$, the system is said to be $p$-moment stable.

Remark II.1. The notion of practical stability will be useful in the case where the diffusion component of the stochastic control system does not vanish at the origin.

Definition II.3 ([18]). A function $V$ is said to be a stochastic input-to-state stable Lyapunov function with respect to an error $e(t)$ if there exist class $\mathcal{K}_{\infty}$ functions $\underline{\alpha}$ and $\bar{\alpha}$ and class $\mathcal{K}$ functions $\alpha$ and $\lambda$ such that

$$
\begin{gathered}
\underline{\alpha}(|x|) \leq V(x) \leq \bar{\alpha}(|x|) \\
\mathcal{L} V(x, t) \leq \lambda(|e|)-\alpha(|x|) .
\end{gathered}
$$

\section{B. Problem Statement}

We consider the state-feedback controlled system (1) with sample-and-hold state measurements, i.e.,

$$
\begin{aligned}
& \mathrm{d} x(t)=f(x, u) \mathrm{d} t+g(x, u) \mathrm{d} w \\
& u(t)=k\left(x_{i}\right), \quad t \in\left[t_{i}, t_{i+1}\right)
\end{aligned}
$$

where $t_{i}, i=1,2, \ldots$ is a sequence of sampling, or triggering, times, and $x_{i}=x\left(t_{i}\right), i=1,2, \ldots$, is the corresponding sequence of measurements of the system state. Let us define the error signal $e(t)$ as

$$
e(t)=x_{i}-x(t), \quad t \in\left[t_{i}, t_{i+1}\right) .
$$

Then (5) is

$$
\mathrm{d} x(t)=f(x, k(x+e)) \mathrm{d} t+g(x, k(x+e)) \mathrm{d} w
$$

The goal of our work is to develop an update rule for the stochastic system (7)-(8) that will render the system practically stable while guaranteeing strictly positive interexecution times $\tau_{i}=t_{i+1}-t_{i}, i=1,2, \ldots$, that is, there is some minimum time between sampling time points. For the purpose of motivating the sequel, we will first begin to formulate the problem using an approach found in literature for deterministic event-triggered and self-triggered control systems and describe how this fails when applied to stochastic control systems.

\footnotetext{
${ }^{1}$ To be concise, note that $p-$ th moment actually refers to the expected value of the Euclidean norm raised to the $p$-th power [16].
}

Along these lines, let us assume the existence of a stochastic input-to-state Lyapunov function satisfying (3)-(4). If we were further to assume that the error were to satisfy [3]

$$
\lambda(|e|) \leq K_{1} \alpha(|x|), \quad 0<K_{1}<1,
$$

then by (4), the Lyapunov function $V$ will decrease since

$$
\mathcal{L} V(x, t) \leq-\left(1-K_{1}\right) \alpha(|x|) .
$$

A condition of the form (9) can be used to implicitly define the sequence of times $\left\{t_{i}\right\}$. For example, a triggering condition of the form

$$
\lambda(|e(t)|)=K_{1} \alpha(|x(t)|) \quad \Rightarrow \quad t_{i+1}:=t
$$

would give rise to an event-triggered framework, since the update rule is based on the current state of the system $x(t)$ and the previous measurement $x_{i}$. In order to show that task periods $\tau_{i}$ are bounded strictly away from zero, the standard technique in literature is to examine the duration for which the error $e(t)$ satisfies a condition like (9) [3, 11]. However, in the stochastic case considered in this work, the error may exceed this bound instantaneously, that is, for any $M<\infty$ and time $t>0$, the Euclidean norm of a solution $e(t)$ to a stochastic differential equation will exceed the level $M$ with non-zero probability, or $\operatorname{Pr}(|e(t)| \geq M)>0$ [15, Exercise 8.13]. Because of this, instead of the sample paths $e(t)$ and $x(t)$, we consider here the $p$-th moments of these processes, $\mathbb{E}\left(|e|^{p}\right)$ and $\mathbb{E}\left(|x|^{p}\right)$, and develop a triggering condition based on these statistics to guarantee stability of $x(t)$ in the $p$-th moment [16].

As a consequence of this basis for the update rule, we must rule out an event-triggered implementation. This is because the controller can only measure an individual sample path of the process $x(t)$, and not the moments of $\mathbb{E}\left(|e|^{p}\right)$ and $\mathbb{E}\left(|x|^{p}\right)$. However, these moments can be predicted on the interval $\left[t_{i}, t_{i+1}\right)$ based on the last-sampled state $x_{i}$, which is suitable for the self-triggered approach and the definition of a rule for the next sample time $t_{i+1}$.

The rest of this paper is devoted to developing a relation involving the sequence of times $\left\{t_{i}\right\}$ and the measurements $x_{i}$ that may be used in a self-triggered control implementation for state-feedback control of the stochastic system (7)(8).

Since this work deals with stability in the $p$-th moment, we must first revise the preliminary notion of stability used to motivate this work (3)-(4) and provide a Lyapunov functionbased stability criterion for $p$-moment stability. We begin with a theorem for $p$-moment ISS.

Theorem II.1. Suppose there exist a convex class $\mathcal{K}_{\infty}$ function $\underline{\alpha}$, class $\mathcal{K}_{\infty}$ functions $\bar{\alpha}$ and $\alpha$, and twice-differentiable function $V(x, t)$ such that

$$
\begin{array}{r}
\alpha\left(|x|^{p}\right) \leq V(x, t) \leq \bar{\alpha}\left(|x|^{p}\right), \\
\mathbb{E} \mathcal{L} V(x, t) \leq \mathbb{E}(\lambda(|e|))-\mathbb{E}(\alpha(|x|))
\end{array}
$$

for all $t \geq 0$, where $\lim _{|x| \rightarrow \infty} \alpha(|x|) / \bar{\alpha}\left(|x|^{p}\right)>0$. Then the system (7)-(8) is p-moment ISS. 
Proof. We refer to the proof of [17, Theorem 3.1], which applies to more general to stochastic retarded systems with Markovian switching, but serves as a proof of Theorem II.1 in the specific case of one discrete state and zero delay. A detailed proof is omitted here and will be presented in another venue.

\section{TRIGGERING CONDITION}

Suppose that the error were to satisfy (cf. (9)):

$$
\mathbb{E}(\lambda(|e|)) \leq K_{1} \mathbb{E}(\alpha(|x|))+d,
$$

for a constant $0<K_{1}<1$, so that

$$
\mathbb{E} \mathcal{L} V(x, t) \leq-\left(1-K_{1}\right) \mathbb{E}(\alpha(|x|))+d,
$$

which would mean that the system is practically $p$-moment stable with a stability margin of $\gamma(d)$ (and $p$-moment stable if $d=0$ ). The following theorem and corollary provide relations that can be used to calculate a strictly positive interexecution time $\tau_{i}=t_{i+1}-t_{i}$ as a function of the norm of lastobserved state $\left|x_{i}\right|$, i.e., in a self-triggered implementation.

Theorem III.1. Assume that in addition to the conditions of Theorem II.1, we assume Lipschitz continuity of the drift and diffusion scaling factor of (1), where, for the drift component, we have the relation

$$
\begin{aligned}
& \left|f(x, k(x+e))-f\left(x^{\prime}, k\left(x^{\prime}+e^{\prime}\right)\right)\right|^{2} \\
& \leq K_{2}\left(\left|x-x^{\prime}\right|^{2}+\left|e-e^{\prime}\right|^{2}\right), \quad \forall x^{\prime} \neq x, e^{\prime} \neq e,
\end{aligned}
$$

and we assume linear growth for the diffusion scaling factor:

$$
|g(x, k(x+e))|^{2} \leq K_{3}\left(1+|x|^{2}+|e|^{2}\right), \quad \forall x, e
$$

for positive constants $K_{2}$ and $K_{3}$. Let us define the increasing functions

$$
\begin{aligned}
& A\left(\tau_{i}\right)=9 \tau_{i}\left(K_{2} \tau_{i}+K_{3}\right) \exp \left(12 \tau_{i}\left(K_{2} \tau_{i}+K_{3}\right)\right), \\
& B\left(\tau_{i}\right)=3 K_{3} \tau_{i} \exp \left(12 \tau_{i}\left(K_{2} \tau_{i}+K_{3}\right)\right),
\end{aligned}
$$

and assume that the inter-execution times satisfy for $t \in$ $\left[t_{i}, t_{i}+\tau_{i}\right)$

$$
\begin{aligned}
& \lambda_{v}\left(A\left(\tau_{i}\right)\left|x_{i}\right|^{2}+B\left(\tau_{i}\right)\right) \\
& \quad \leq K_{1} \alpha_{c}\left(\left(\frac{1}{3}-A\left(\tau_{i}\right)\right)\left|x_{i}\right|^{2}-B\left(\tau_{i}\right)+d_{\alpha}\right),
\end{aligned}
$$

for a constant $d_{\alpha} \geq 0$, a convex class $\mathcal{K}$ function $\alpha_{c}(\cdot)$ and a concave class $\mathcal{K}$ function which satisfy

$$
\begin{aligned}
& \alpha_{c}\left(2|x|^{2}\right) \leq 2 \alpha(|x|) \\
& \lambda_{v}(|e|) \geq \lambda(\sqrt{|e|}) .
\end{aligned}
$$

Then (14) will hold with $d=K_{1} \alpha_{c}\left(2 d_{\alpha}\right) / 2$. Moreover, the execution times do not reach an accumulation point, i.e., $\tau_{i}>0$.

Remark III.1. The inter-execution times $\tau_{i}, i=0,1, \ldots$, may be solved numerically from (20) based on the norm of the last-observed state $\left|x_{i}\right|$.
Proof. Before addressing the inter-execution times, we instead start with a more conservative condition than (20), namely,

$$
\lambda_{v}\left(\mathbb{E}\left(|e|^{2}\right)\right) \leq K_{1} \alpha_{c}\left(\mathbb{E}\left(|x|^{2}\right)+d_{\alpha}\right)
$$

We show that this implies (14), and then prove that (23) is satisfied when the assumption (20) holds.

Beginning with the right hand side of (23), since $\alpha_{c}(\cdot)$ is convex, we have from (21) and Jensen's inequality that

$$
\begin{aligned}
K_{1} \alpha_{c} & \left(\mathbb{E}\left(|x|^{2}\right)+d_{\alpha}\right) \\
& =K_{1} \alpha_{c}\left(2\left(\frac{1}{2} \mathbb{E}\left(|x|^{2}\right)+\frac{1}{2} d_{\alpha}\right)\right) \\
& \leq K_{1} \mathbb{E}\left(\alpha_{c}\left(2|x|^{2}\right) / 2\right)+K_{1} \alpha_{c}\left(2 d_{\alpha}\right) / 2 \\
& \leq K_{1} \mathbb{E}(\alpha(|x|))+d,
\end{aligned}
$$

where $d=K_{1} \alpha_{c}\left(2 d_{\alpha}\right) / 2$. Through the concavity of $\lambda_{v}(\cdot)$, the left hand side of (23) can also be made to match that of (14) using Jensen's inequality and (22).

Next, we find an upper bound on $\mathbb{E}\left(|e|^{2}\right)$ during the interval $\left[t_{i}, t_{i}+\tau_{i}\right)$ in terms of the inter-execution time $\tau_{i}$ and the last-observed state $\left|x_{i}\right|^{2}$. Recall that from (5) and (7), the error kinematics satisfy for $t \in\left[t_{i}, t_{i+1}\right)$ :

$$
e(t)=-\int_{t_{i}}^{t} f(x, u) \mathrm{d} s-\int_{t_{i}}^{t} g(x, u) \mathrm{d} w_{s} .
$$

Then for any $t \in\left[t_{i}, t_{i+1}\right)$,

$$
\begin{aligned}
\mathbb{E}\left(|e|^{2}\right) & =\mathbb{E}\left(\left|\int_{t_{i}}^{t} f(x, u) \mathrm{d} s\right|^{2}\right)+\mathbb{E}\left(\left|\int_{t_{i}}^{t} g(x, u) \mathrm{d} w_{s}\right|^{2}\right) \\
+ & 2 \mathbb{E}\left(\left(\int_{t_{i}}^{t} f(x, u) \mathrm{d} s\right)^{\top}\left(\int_{t_{i}}^{t} g(x, u) \mathrm{d} w_{s}\right)\right) \\
\leq & 3 \mathbb{E}\left(\left|\int_{t_{i}}^{t} f(x, u) \mathrm{d} s\right|^{2}\right)+3 \mathbb{E}\left(\left|\int_{t_{i}}^{t} g(x, u) \mathrm{d} w_{s}\right|^{2}\right) .
\end{aligned}
$$

Using the Itō Isometry [15] and (16)-(17), we can obtain

$$
\begin{aligned}
\mathbb{E}\left(|e|^{2}\right) \leq 3\left(t_{i+1}-t_{i}\right) \mathbb{E}\left(\int_{t_{i}}^{t}|f(x, u)|^{2} \mathrm{~d} s\right) \\
+3 \mathbb{E}\left(\int_{t_{i}}^{t}|g(x, u)|^{2} \mathrm{~d} s\right) \\
\leq 3\left(t_{i+1}-t_{i}\right) \mathbb{E}\left(\int_{t_{i}}^{t} K_{2}\left(|x|^{2}+|e|^{2}\right) \mathrm{d} s\right) \\
\quad+3 \mathbb{E}\left(\int_{t_{i}}^{t} K_{3}\left(1+|x|^{2}+|e|^{2}\right) \mathrm{d} s\right) \\
=3 K_{3}\left(t_{i+1}-t_{i}\right)+3\left(\left(t_{i+1}-t_{i}\right) K_{2}+K_{3}\right) \int_{t_{i}}^{t} \mathbb{E}\left(|x|^{2}\right) \mathrm{d} s \\
\quad+3\left(\left(t_{i+1}-t_{i}\right) K_{2}+K_{3}\right) \int_{t_{i}}^{t} \mathbb{E}\left(|e|^{2}\right) \mathrm{d} s,
\end{aligned}
$$


or, since $|x|^{2} \leq 3\left|x_{i}\right|^{2}+3|e|^{2}$,

$$
\begin{aligned}
\mathbb{E}\left(|e|^{2}\right) \leq & 3 K_{3}\left(t_{i+1}-t_{i}\right) \\
& +9\left(\left(t_{i+1}-t_{i}\right) K_{2}+K_{3}\right) \int_{t_{i}}^{t} \mathbb{E}\left(\left|x_{i}\right|^{2}\right) \mathrm{d} s \\
& +12\left(\left(t_{i+1}-t_{i}\right) K_{2}+K_{3}\right) \int_{t_{i}}^{t} \mathbb{E}\left(|e|^{2}\right) \mathrm{d} s \\
\leq & C\left(\left|x_{i}\right|^{2}, \tau_{i}\right),
\end{aligned}
$$

where we have used Gronwall's inequality, and $C\left(\left|x_{i}\right|^{2}, \tau_{i}\right)=A\left(\tau_{i}\right)\left|x_{i}\right|^{2}+B\left(\tau_{i}\right)$ with $A\left(\tau_{i}\right)$ and $B\left(\tau_{i}\right)$ defined in (18)-(19). Substituting (26) in the condition (23),

$$
\lambda_{v}\left(C\left(\left|x_{i}\right|^{2}, \tau_{i}\right)\right) \leq K_{1} \alpha_{c}\left(\mathbb{E}\left(|x|^{2}\right)+d_{\alpha}\right),
$$

or, since $\left|x_{i}\right|^{2}=\mathbb{E}\left(|x+e|^{2}\right) \leq 3 \mathbb{E}\left(|x|^{2}\right)+3 \mathbb{E}\left(|e|^{2}\right) \leq$ $3 \mathbb{E}\left(|x|^{2}\right)+3 C\left(\left|x_{i}\right|^{2}, \tau_{i}\right)$, the right hand side can be made more conservative,

$$
\lambda_{v}\left(C\left(\left|x_{i}\right|^{2}, \tau_{i}\right)\right) \leq K_{1} \alpha_{c}\left(\frac{\left|x_{i}\right|^{2}}{3}-C\left(\left|x_{i}\right|^{2}, \tau_{i}\right)+d_{\alpha}\right),
$$

which is exactly assumption (20).

Next, to show the existence of a lower bound for the inter-execution times implicitly defined by (20) or (28), we point out that $C(\cdot, \cdot)$ is increasing in both arguments, and, consequently, for (28) to admit a $\tau_{i}>0$ for any $\left|x_{i}\right|^{2}$, then (28) should hold when the last-observed state approaches $\left|x_{i}\right|^{2}=0$. Letting $\left|x_{i}\right|^{2}=0$ in (28), we have

$$
0<\alpha_{c}^{-1}\left(\frac{\lambda_{v}\left(B\left(\tau_{i}\right)\right)}{K_{1}}\right)+B\left(\tau_{i}\right)<d_{\alpha},
$$

and so, since $B\left(\tau_{i}\right)$ is an increasing function, $\tau_{i}>0$.

If $d_{\alpha}=0(d=0)$ in (28), we would need $C\left(\left|x_{i}\right|^{2}, \tau_{i}\right)<$ $\left|x_{i}\right|^{2} / 3$ in order to keep a positive right hand side in (28). As the last-observed state approaches the origin, however, some systems may still allow for positive inter-execution times for any $\left|x_{i}\right|$ and $d_{\alpha}=0$, based on the following corollary.

Corollary III.1. Suppose that $g(\cdot, \cdot)$ also admits an equilibrium point $g(0,0)=0$, which, by a Lipschitz argument similar to (16), allows us to write

$$
|g(x, k(x+e))|^{2} \leq K_{3}\left(|x|^{2}+|e|^{2}\right) .
$$

If the inter-execution time $\tau_{i}$ is such that

$$
A\left(\tau_{i}\right) \leq \kappa / 3
$$

for a constant $0<\kappa<1$ satisfying

$$
\lambda_{v}\left(\frac{\kappa}{3}\left|x_{i}\right|^{2}\right) \leq K_{1} \alpha_{c}\left(\frac{1}{3}(1-\kappa)\left|x_{i}\right|^{2}\right),
$$

then condition (23) with $d_{\alpha}=0$ implies (14) with $d=0$, i.e., p-moment stability is guaranteed, with $\tau_{i}>0$.

Proof. Using (29) instead of (17) in the proof of Theorem III.1, we can derive (28) with $C\left(\left|x_{i}\right|^{2}, \tau_{i}\right)=A\left(\tau_{i}\right)\left|x_{i}\right|^{2}$. Substituting (30) into (31), we obtain (28) with $d_{\alpha}=0$, which implies (14) with $d=0$.

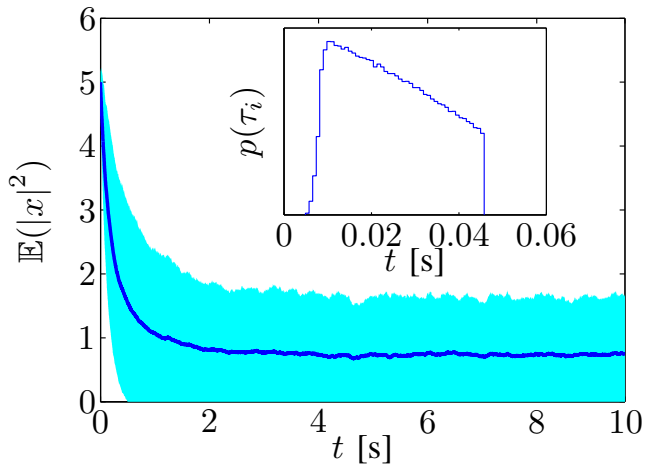

Fig. 1. Evolution of the second moment $\mathbb{E}\left(|x|^{2}\right)$ over a 10 second simulation, averaged over 1000 sample trajectories, with standard deviation bands shown. The initial condition is a vector of magnitude $x_{1}(0)^{2}+$ $x_{2}(0)^{2}=5$ and random direction. Since the noise does not vanish, we expect to achieve practical $p$-moment stability. Inset: Distribution of interexecution times $p\left(\tau_{i}\right), \mathbb{E}\left(\tau_{i}\right)=0.0245, \min \left(\tau_{i}\right)=0.0049$.

Note that this additional assumption to allow for $d=0$ results in a periodic triggering condition (30), which may be solved numerically, as before. In other words, when the diffusion vanishes at the origin and we are tasked with $p$-moment stability, the self-triggering rule (20) should be replaced by the periodic triggering rule (30) when $x_{i}$ approaches the origin.

\section{EXAMPLES}

In this section we provide a pair of examples in which the noise is either constant or vanishing at the origin.

\section{A. Nonlinear example with constant noise intensity}

The first example is a nonlinear system whereby the state $x=\left[x_{1}, x_{2}\right]^{\top}$ obeys

$$
\begin{aligned}
& \mathrm{d} x_{1}=\left(-x_{1}+x_{2}\right) \mathrm{d} t+\sigma_{1} \mathrm{~d} w_{1} \\
& \mathrm{~d} x_{2}=\left(u(x) \sin \left(x_{1}\right)-4 x_{2}\right) \mathrm{d} t+\sigma_{2} \mathrm{~d} w_{2}
\end{aligned}
$$

with $u(x)=x_{1}+x_{2}$, and where the noise intensity $\sigma_{1}=$ $\sigma_{2}=1$. Let $V(x)=\left(x_{1}^{2}+x_{2}^{2}\right) / 2$. Then

$$
\begin{aligned}
& \mathcal{L} V=x_{1}\left(-x_{1}+x_{2}\right) \\
& +x_{2}\left(\left(x_{1}+x_{2}+e_{x_{1}}+e_{x_{2}}\right) \sin \left(x_{1}\right)-4 x_{2}\right)+\frac{1}{2}\left(\sigma_{1}^{2}+\sigma_{2}^{2}\right) \\
& \leq-x_{1}^{2}+2\left|x_{1}\right|\left|x_{2}\right|-3 x_{2}^{2}+\left|e_{x_{1}}+e_{x_{2}}\right|\left|x_{2}\right| \\
& \leq-\left[\left|x_{1}\right| \quad\left|x_{2}\right|\right] Q\left[\begin{array}{l}
\left|x_{1}\right| \\
\left|x_{2}\right|
\end{array}\right]+3|e|^{2}+\sigma^{2} \\
& \leq-\delta|x|^{2}+3|e|^{2}+d,
\end{aligned}
$$

where $\delta \approx 0.38$ is the smallest eigenvalue of $Q=$ $\left[\begin{array}{cc}1 & -1 \\ -1 & 2\end{array}\right]$, and where $d=1=K_{3}$. Taking the expected value of both sides of (34),

$$
\mathbb{E} \mathcal{L} V \leq-\delta \mathbb{E}\left(|x|^{2}\right)+3 \mathbb{E}\left(|e|^{2}\right)+d,
$$

and so we choose $K_{1}=0.125<\delta / 3$, where $\lambda_{v}(|e|)=|e|$ and $\alpha_{c}(|x|)=|x|$, so that $d_{\alpha}=d / K_{1}$. Then with $K_{2}=3$, 


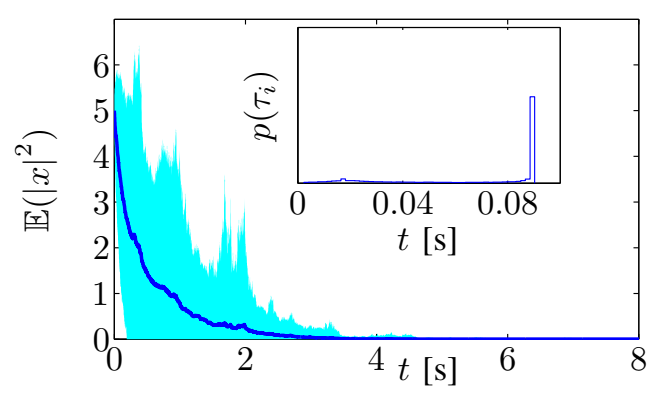

Fig. 2. Evolution of the second moment $\mathbb{E}\left(|x|^{2}\right)$ over an 8 second simulation, averaged over 500 sample trajectories, with standard deviation bands shown. The initial condition is a vector of magnitude $x_{1}(0)^{2}+x_{2}(0)^{2}=5$ and random direction. (Inset) Distribution of inter-execution times $p\left(\tau_{i}\right)$ before $t=7.5 \mathrm{~s}, \mathbb{E}\left(\tau_{i}\right)=0.0691, \min \left(\tau_{i}\right)=0.0024$ (or $\mathbb{E}\left(\tau_{i}\right)=0.0039$ and $\min =9.86 \times 10^{-4}$ when times $t>7.5 \mathrm{~s}$ are included).

we require from (28)

$$
C\left(\left|x_{i}\right|^{2}, \tau_{i}\right) \leq K_{1}\left(\frac{1}{3}\left|x_{i}\right|^{2}-C\left(\left|x_{i}\right|^{2}, \tau_{i}\right)+d_{\alpha}\right)
$$

in order to guarantee practical stability in the $p=2$ moment. Figure 1 shows $\mathbb{E}\left(|x|^{2}\right)$ based on 1000 simulations along with the distribution of inter-execution times $\tau_{i}$.

\section{B. Nonlinear example with vanishing noise}

Next consider the same model as (32)-(33), but with statedependent noise intensities $\sigma_{1}=x_{1}$ and $\sigma_{2}=x_{2}$. We can now obtain (34) with $d=0$ and $\delta \approx 0.086$, and $K_{1}=$ $0.028<\delta / 3, K_{2}=2$, and $K_{3}=1$. With $\lambda_{v}(|e|)=|e|$ and $\alpha_{c}(|x|)=|x|$, we can solve for $\kappa$ in (31) as $\kappa<K_{1} /(1+$ $\left.K_{1}\right)=0.0278$, and we choose $\kappa=0.027$. A periodic control update using the triggering times determined by (30) leads to $\tau_{i}=9.86 \times 10^{-4}$. Therefore, to speed up convergence, we first aim for practical stability with $d_{\alpha}=1$, which allows $\tau_{i}$ 's on the order of 0.02 based on the self-triggering condition (28), for the first 7.5 seconds of the $10 \mathrm{~s}$ simulation, before switching to a periodic update from (30). In Fig. 2, it is seen that $\mathbb{E}\left(|x|^{2}\right)$ is stabilized to the origin, but this happens before $7.5 \mathrm{~s}$, which indicates that (30) may have room for improvement.

\section{CONCLUSIONS}

This paper presents a self-triggered control scheme for state-feedback controlled stochastic differential equations. Since the inequality-based sampling conditions found in previous event- and self-triggered control works may be instantaneously violated in the presence of the stochastic noise considered in this paper, we instead focus on the moments of the state distribution. These moments can be predicted based on the last-observed state and are used here to develop a self-triggered control scheme. The scheme is shown to produce strictly positive inter-execution times that guarantee $p$-moment stability of the process.

In future work, a less conservative sampling rule may be obtainable by drawing from the various approaches in literature for the deterministic case that lengthen the interexecution times. Moreover, the robustness of our scheme to a delay between state sampling and implementation of the updated control, i.e., a task delay, will be examined. The application of this scheme for stochastic problems where control updates or state sampling are expensive or limited, e.g., multi-agent robotic systems and distributed estimation, will also be the focus of future work.

\section{ACKNOWLEDGMENT}

This work is supported by NSF GRFP No. DGE-0809125 and by NSF/Vetenskapsrådet Nordic Research Opportunity. The authors thank the reviewers for their feedback.

\section{REFERENCES}

[1] K. Årzén, "A simple event-based PID controller," in Proceedings of the 14th IFAC World Congress, Beijing, China, 1999.

[2] K. Astrom and B. Bernhardsson, "Comparison of Riemann and Lebesgue sampling for first order stochastic systems," in Proceedings of the 41st IEEE Conference on Decision and Control, Las Vegas, NV, 2002, pp. 2011-2016.

[3] P. Tabuada, "Event-Triggered Real-Time Scheduling of Stabilizing Control Tasks," IEEE Transactions on Automatic Control, vol. 52, no. 9, pp. 1680-1685, 2007.

[4] R. Postoyan, A. Anta, D. Nesic, and P. Tabuada, "A unifying Lyapunov-based framework for the event-triggered control of nonlinear systems," in Proceedings of the 50th IEEE Conference on Decision and Control and European Control Conference, Orlando, FL, 2011, pp. 2559-2564.

[5] X. Wang and M. Lemmon, "Event design in event-triggered feedback control systems," in Proceedings of the 47th IEEE Conference on Decision and Control, Cancun, Mexico, 2008, pp. 2105-2110.

[6] A. Anta and P. Tabuada, "Self-triggered stabilization of homogeneous control systems," in Proceedings of the 2008 American Control Conference, Seattle, WA, Jun. 2008, pp. 4129-4134.

[7] M. Lemmon, T. Chantem, X. S. Hu, and M. Zyskowski, "On selftriggered full-information H-infinity controllers," in Proceedings of the 10th International Workshop on Hybrid Systems: Computation and Control, Pisa, Italy, 2007, pp. 371-384.

[8] M. Mazo and P. Tabuada, "On event-triggered and self-triggered control over sensor/actuator networks," in Proceedings of the 47th IEEE Conference on Decision and Control, Cancun, Mexico, 2008, pp. $435-440$.

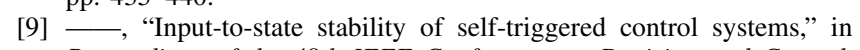
Proceedings of the 48th IEEE Conference on Decision and Control, Shanghai, China, 2009, pp. 928-933.

[10] M. Velasco, P. Marti, and J. Fuertes, "The self triggered task model for real-time control systems," in Work in Progress Proceedings of the 24th IEEE Real-Time Systems Symposium, Cancun, Mexico, 2003, pp. 67-70.

[11] X. Wang and M. D. Lemmon, "Self-triggered Feedback Control Systems with Finite-Gain L2 Stability," IEEE Transactions on Automatic Control, vol. 54, no. 3, pp. 452-467, 2009.

[12] _ , "Self-Triggering Under State-Independent Disturbances," IEEE Transactions on Automatic Control, vol. 55, no. 6, pp. 1494-1500, Jun. 2010.

[13] D. V. Dimarogonas, E. Frazzoli, and K. H. Johansson, "Distributed self-triggered control for multi-agent systems," in Proceedings of the 49th IEEE Conference on Decision and Control, Atlanta, GA, Dec. 2010, pp. 6716-6721.

[14] E. D. Sontag, "Input to state stability: Basic concepts and results," in Nonlinear and Optimal Control Theory, P. Nistri and G. Stefani, Eds. Berlin: Springer-Verlag, 2008, ch. 3, pp. 163-220.

[15] B. Oksendal, Stochastic Differential Equations: An Introduction with Applications, 6th ed. Berlin: Springer-Verlag, 2003.

[16] X. Mao, Stochastic Differential Equations and Applications, 2nd ed. Chichester, UK: Horwood Publishing, 2012.

[17] L. Huang and X. Mao, "On input to state stability of stochastic retarded systems with Markovian switching," IEEE Transactions on Automatic Control, vol. 54, no. 8, pp. 1898-1902, 2009.

[18] S.-j. Liu, J.-f. Zhang, and Z.-p. Jiang, "A Notion of Stochastic Input-toState Stability and Its Application to Stability of Cascaded Stochastic Nonlinear Systems," Acta Mathematicae Applicatae Sinica, English Series, vol. 24, no. 1, pp. 141-156, 2008. 